\title{
Area and Power Efficient Ultra-Wideband Transmitter Based on Active Inductor
}

\author{
Kerim Ture, Student Member, IEEE, Arnout Devos, Student Member, IEEE, Franco Maloberti, Life Fellow, IEEE, \\ and Catherine Dehollain, Member, IEEE
}

\begin{abstract}
This paper presents the design of an impulse radio ultra-wideband (IR-UWB) transmitter for low-power, shortrange, and high-data rate applications such as high density neural recording interfaces. The IR-UWB transmitter pulses are generated by modulating the output of a local oscillator. The large area requirement of the spiral inductor in a conventional on-chip LC tank is overcome by replacing it with an active inductor topology. The circuit has been fabricated in a UMC CMOS $180 \mathrm{~nm}$ technology, with a die area of $0.012 \mathrm{~mm}^{2}$. The temporal width of the output waveform is determined by a pulse generator based on logic gates. The measured pulse is compliant with Federal Communications Commission (FCC) power spectral density limits and within the frequency band of 3-6 GHz. For the minimum pulse duration of $1 \mathrm{~ns}$, the energy consumption of the design is $20 \mathrm{pJ}$ per bit, while transmitting at a $200 \mathrm{Mbps}$ data rate with an amplitude of $130 \mathrm{mV}$.
\end{abstract}

Index Terms-Wireless data transmission, low-power, highdata rate, impulse radio ultra-wideband (IR-UWB) transmitter, pulse generation, active inductor.

\section{INTRODUCTION}

$\mathbf{R}$ ECORDING neural activities has an essential role in various applications such as brain-machine interfaces (BMI) and monitoring neurological disorders like epilepsy, Alzheimer and Parkinson diseases. In recent years, several implanted neural recordings systems [1]-[6] have been investigated to play an important role in the treatment of neurological diseases.

Thanks to the improvements in electrodes, sensors, and microelectronics, the need for quality and quantity of extracted information has been on the rise. Recording systems are expected to perform better regarding spatial and temporal resolutions. Those demands drive the technology to implanted systems with new challenges such as power management and wireless communication. Such fully implanted systems require being powered by a battery. This can be achieved by remote powering, an energy harvesting mechanism or a combination of both such that operation for an extended amount of time is guaranteed, without the need for another surgery. The implanted electronics must have a low power implementation because of limited energy resources. More importantly, the minimization of temperature elevation in the surrounding tissues is necessary to avoid damaging them [7].

This work has been supported by the Swiss NSF under grant number 200021-157090.

K. Ture, A. Devos and C. Dehollain are with the RFIC Group, Ecole Polytechnique Fédérale de Lausanne, 1015 Lausanne, Switzerland (e-mail: kerim.ture@epfl.ch).

F. Maloberti is with the Department of Electrical, Computer, and Biomedical Engineering, University of Pavia, 27100 Pavia, Italy.
To address the high spatial and temporal resolution requirements, microelectrode arrays have been developed for simultaneous and high-density monitoring of action potentials in distributed brain areas [8]. Data acquired from hundreds of electrodes can quickly reach up to hundreds of megabits per second (Mbps). Those constraints obligate wireless transmitters with high data-rate at low power dissipation for neural monitoring applications. In recent years, several implanted neural monitoring systems have been developed to enable wireless neural activity recording. For example, in the 64channel wireless micron-scale electrocorticography $(\mu \mathrm{ECoG})$ solution presented in [9], Miller-encoded serialized data is transmitted at $1 \mathrm{Mbps}$ thanks to a backscattering modulator. As another example, a binary phase-shift-keying (BPSK) modulated transmitter at $2.4 \mathrm{GHz}$ with the capability of delivering data up to 8 Mbps from 8 channels was developed [10]. Further, a neuroelectrical monitoring CMOS system utilized a Manchester-encoded FSK modulation scheme with a carrier frequency at $916.4 \mathrm{MHz}$ and data rate of $1.5 \mathrm{Mbps}$ across a distance of $10 \mathrm{~m}$ [11]. Recently, an on-off keying (OOK) modulated transmitter at $430 \mathrm{MHz}$ with a $12 \mathrm{Mbps}$ data rate was verified in in-vivo experiments for epilepsy monitoring [12].

Higher data rates can be achieved by using pulse-based communication methods instead of carrier-based radios at the cost of a more limited transmission range. In this kind of wideband data transmission, each symbol is encoded by a train of short pulses. Kiani et al. have developed a nearfield data transmission across inductive telemetry links based on pulse harmonic modulation with a data rate of $20 \mathrm{Mbps}$ [13]. Impulse radio ultra-wideband (IR-UWB) technology has drawn attention for low power, high data rate communication for implanted systems [14]-[16]. There are three main methods for generating UWB pulses: combining filtered edges [14], combining pulses [15] and modulating a local oscillator [16]. The filtered combined edges method is desirable for its low power consumption. However, generating FCC compliant pulses requires an area expensive filter implementation [14]. Another low power IR-UWB method combines short pulses using highly digital circuits. However, due to the significant effect of variations between stages on pulse duration and its power spectral density (PSD), this method requires complicated calibrations [17]. A ring oscillator can be used as the local oscillator (LO) to be modulated for pulse generation, but it suffers from instability in frequency. The drawback of LC oscillators as an alternative to ring oscillators is the higher silicon area consumption and power requirements [18].

It has been shown in [19] that a Gaussian pulse shape 


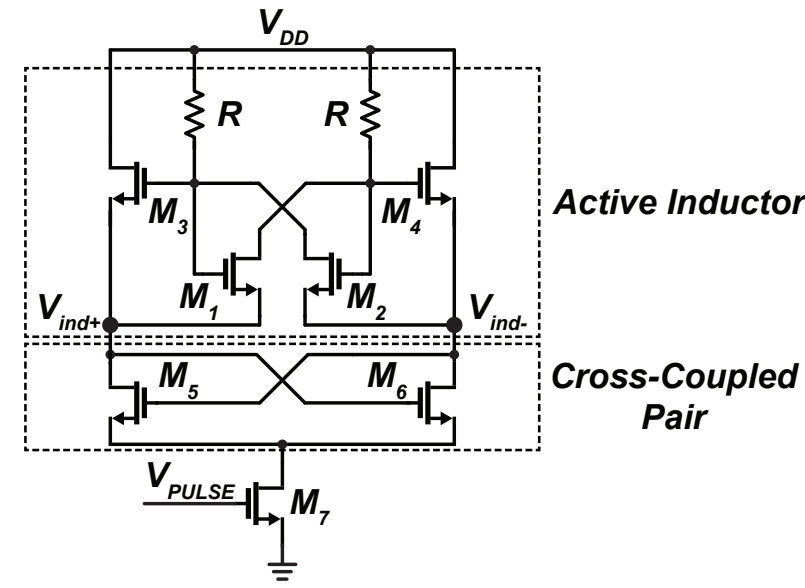

Fig. 1. Schematic of the proposed pulsed active inductor oscillator.

optimizes for maximum bandwidth and minimum sidelobe leakage to fulfill FCC limits. However, Gaussian pulses require complex circuit design and high power consumption. Triangular shaped pulses can be seen as a favorable alternative, considering the design simplicity, power and area requirements.

In this paper, we present an energy and area efficient, high data rate UWB transmitter based on the modulation of an LO employing an active inductor. Using an active inductor topology overcomes the area limitation caused by the passive inductor in a conventional LC tank while at the same time reducing the power consumption. Short pulses with a triangular shape envelope are generated by taking advantage of startup and turnoff periods of the LO. The remainder of this paper is organized as follows: The design of the active inductorbased oscillator is presented in Section II. Section III describes the design of the full UWB transmitter. Section IV shows the measurement results from the fabricated chip. Finally, Section V summarizes the contribution of this work.

\section{Active Inductor BASEd Local Oscillator}

To decrease the large area consumption associated with conventional spiral CMOS integrated inductors while retaining the start-up behavior of a regular LC tank oscillator, an active inductor can be used. An active inductor is built solely out of transistors and capacitors, thus removing the need for a large area integrated inductor. A significant drawback of the usage of active inductors in RF systems is that they have much worse phase noise performance than their large area inductor counterparts for the same power consumption, as shown in [20]. However, since the IR-UWB technique is pulse based, phase noise is of no concern and active inductors become very attractive to construct a low-area low-power inductor as part of the LC tank.

The schematic of the LO based on an active inductor is shown in Fig. 1. The active inductor topology introduced by $\mathrm{Lu}$ et al. [21] is realized by the transistors $M_{1}-M_{4}$ and two resistors R. Drain-source and drain-gate parasitic capacitances of the cross-coupled pair of transistors, $M_{5}$ and $M_{6}$, constitute an $L C$ tank together with the active inductor. Moreover, $M_{5}$ and $M_{6}$ provide a sufficient negative conductance to satisfy

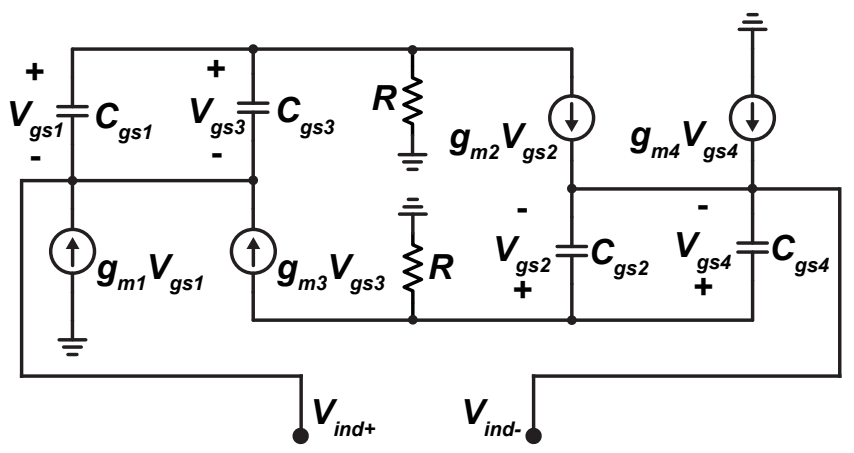

(a)

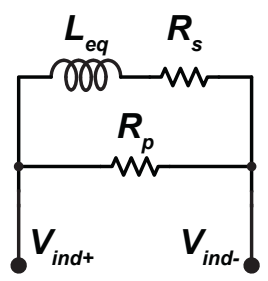

(b)

Fig. 2. (a) Small-signal model of the active inductor. (b) Simplified equivalent model of the active inductor.

the Barkhausen criterion [22] by compensating the loss of the $L C$ tank and ensure startup and sustained oscillation. By introducing a current source at the bottom of the LO topology, the oscillator can be turned ON or OFF. Since an active inductor is used in cooperation with the parasitic capacitances of the transistors, an LC start-up and slow-down behavior can be realized as in [23] without the need for large area integrated passive inductors.

The selection of transistor dimensions, the resistance value, and determination of the oscillation criterion requires a smallsignal analysis. The small-signal model of the active inductor, leaving out the non-dominant drain-source capacitances, and its equivalent model are shown in Figures $2 a$ and $2 b$, respectively. Symmetry in the circuit yields the input impedance as

$$
Z_{i n}=\frac{2\left[j w\left(C_{g s 1}+C_{g s 3}\right) R-g_{m 1} R+1\right]}{g_{m 1}+g_{m 3}+j w\left(C_{g s 1}+C_{g s 3}\right)} .
$$

For two criteria, $2 g_{m 1}+g_{m 3}>R^{-1}$ and $g_{m 1}<R^{-1}$, the parameters in the equivalent model of the active inductor are given by

$$
\begin{aligned}
R_{p} & =2 R \\
R_{s} & =\frac{2 R\left(1-g_{m 1} R\right)}{\left(2 g_{m 1}+g_{m 3}\right) R-1} \\
L_{e q} & =\frac{2\left(C_{g s 1}+C_{g s 3}\right) R^{2}}{\left(2 g_{m 1}+g_{m 3}\right) R-1} .
\end{aligned}
$$

This equivalent model indicates the requirement of the negative conductance provided by the cross-coupled pair of $M_{5}$ and $M_{6}$. The transconductance $g_{m 5}$ needs to be higher than $R_{p}^{-1}$ to guarantee the start-up of the oscillation. Transistor $M_{7}$ is sized such that it provides the necessary bias current to the LO for the desired oscillation and start-up behavior for incoming short pulses to its gate. 


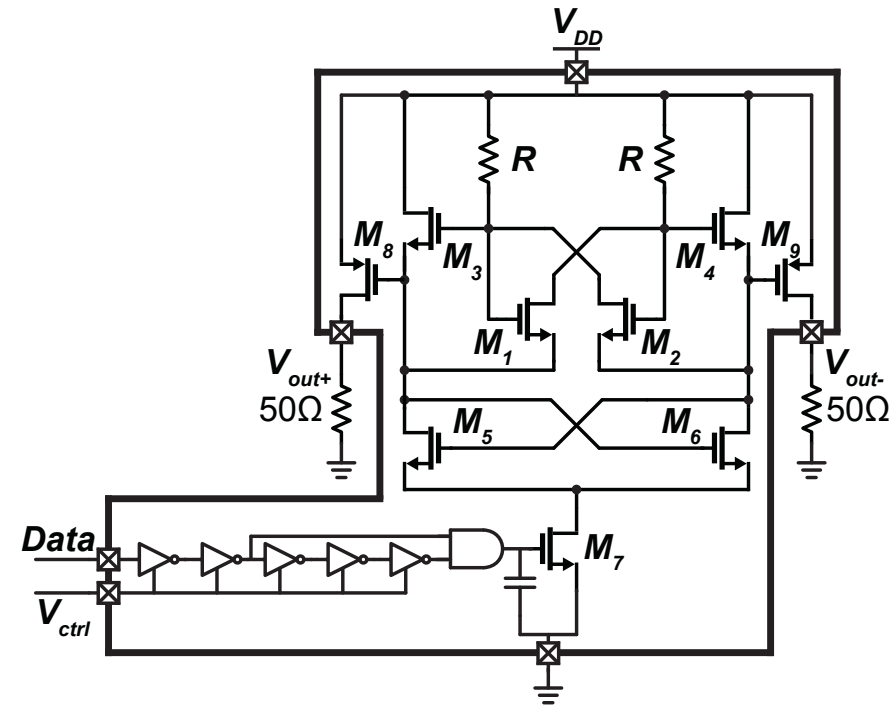

Fig. 3. Proposed IR-UWB transmitter.

TABLE I

CIRCUIT PARAMETERS OF THE LO

\begin{tabular}{cc}
\hline \hline Transistors & $\mathrm{W} / \mathrm{L}(\mu \mathrm{m} / \mu \mathrm{m})$ \\
$M_{1}, M_{2}$ & $25 / 0.18$ \\
$M_{3}, M_{4}$ & $25 / 0.18$ \\
$M_{5}, M_{6}$ & $80 / 0.18$ \\
$M_{7}$ & $150 / 0.18$ \\
\hline \hline
\end{tabular}

\section{UWB TRANSmitter Design}

Fig. 3 shows the proposed IR-UWB transmitter which includes the active inductor-based oscillator discussed in Section II, a pulse generator, and two single stage amplifiers constituted by transistors $M_{8}$ and $M_{9}$ both of size $50 \mu \mathrm{m} / 0.18 \mu \mathrm{m}$. The pulse generator is composed of an inverter chain and an AND-gate . In the inverter chain, current-starved inverters are used to provide the flexibility of controlling transition times and delays by an external voltage, $V_{c t r l}$. This feature provides control over the width of the generated pulse. The AND-gate logic circuit needs to be sized such that the output stage can drive the gate of transistor $M_{7}$ for a desired bias current of the LO. The value of the resistor R is chosen as $125 \Omega$ and the transistor sizes used for the active inductor-based oscillator design are reported in Table I.

The differential output of the oscillator needs to be isolated from the loads to prevent the highly sensitive active inductor to be affected by the load. For isolation of the oscillator and amplification of the output waveform, two single stage amplifiers were utilized, and their drains directly drive the differential loads as shown in Fig 3.

\section{Measurement Results}

The proposed IR-UWB transmitter is fabricated using UMC $180 \mathrm{~nm}$ 1P6M MM/RF process technology. The total active area occupies only $0.15 \times 0.14 \mathrm{~mm}^{2}$. The fabricated chip is shown in Fig. 4, where the IR-UWB transmitter part is

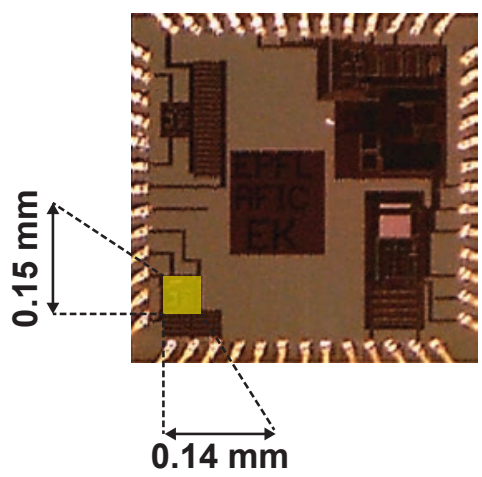

Fig. 4. Microphotograph of the IR-UWB transmitter in $180 \mathrm{~nm}$ CMOS.

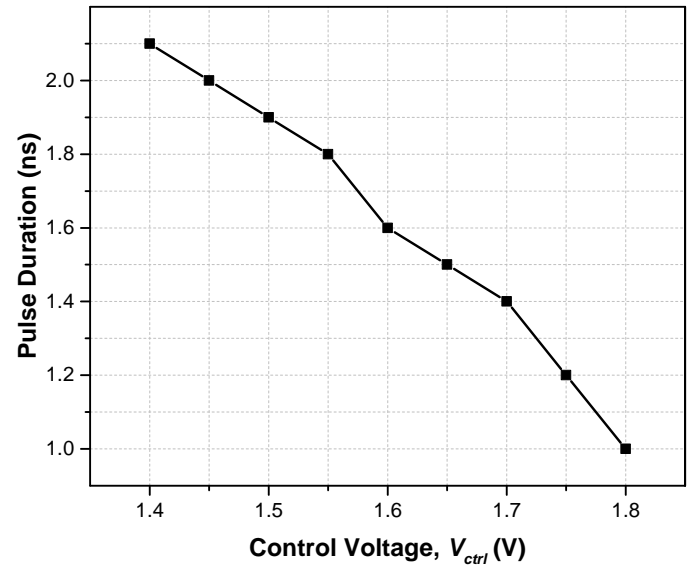

Fig. 5. Duration of the pulse with the control voltage, $V_{c t r l}$.

highlighted. The measurements where carried out on a chipon-board package with a $20 \mathrm{GS} / \mathrm{s}$ oscilloscope.

The pulse width control voltage, $V_{c t r l}$, is swept from $1.4 \mathrm{~V}$ to $1.8 \mathrm{~V}$, and its effect on the pulse width is shown in Fig. 5. Increasing the voltage increases the switching speeds of the inverters in the pulse generator and thus makes the pulse width narrower. The relation between the pulse width and its control voltage is almost linear. Since the output waveform depends on the startup and turnoff periods of the oscillator, the change in the pulse width affects also the amplitude of the output signal. Fig. 6 depicts two examples of output waveforms for different values of $V_{c t r l}$. The generated minimum pulse width creates an oscillation with an amplitude of $130 \mathrm{mV}$. When applying a $200 \mathrm{Mbps}$ data stream, composed by uniformly distributed digital " 1 "s and " 0 "s, the measured energy per pulse of the IR-UWB transmitter is $27.5 \mathrm{pJ}$ and $20 \mathrm{pJ}$, for the waveforms in Fig. 6a and 6b, respectively.

Fig. 7 shows the power spectral densities of the FCCcompliant pulses in Fig. 6. As can be seen in Fig. 7b, the reduction in amplitude and duration of the pulse results in a broader and lower PSD. Since the center frequency is far enough from the lower limit of the FCC mask, the increase in bandwidth does not cause a violation.

The performance of the designed UWB transmitter is sum- 
TABLE II

SUMMARY OF MEASURED PERFORMANCE AND COMPARISON WITH RECENT STUDIES

\begin{tabular}{|c|c|c|c|c|c|c|c|c|c|}
\hline & $\begin{array}{l}\text { Tech. } \\
(\mathbf{n m})\end{array}$ & Modulation & Method & $\begin{array}{c}\text { Frequency } \\
\text { Band } \\
(\mathbf{G H z})\end{array}$ & $\begin{array}{l}\text { Data } \\
\text { Rate } \\
\text { (Mbps) }\end{array}$ & $\begin{array}{c}\text { Pulse } \\
\text { Amplitude } \\
(\mathbf{m V})\end{array}$ & $\begin{array}{r}\text { Energy } \\
(\mathbf{p J} / \mathbf{b})\end{array}$ & $\begin{array}{c}\text { Area } \\
\text { (core) } \\
\left(\mathbf{m m}^{2}\right)\end{array}$ & $\begin{array}{c}\text { Normalized } \\
\text { Energy } \\
\left(\mathbf{m m}^{2} \cdot \mathbf{p J}\right) /(\mathbf{b} . \mathbf{V})\end{array}$ \\
\hline TMTT '17 [24] & 180 & BPSK-PAM & Edge combining & $3.5-6.5$ & 250 & 500 & 86 & 0.22 & 18.92 \\
\hline JSSC '17 [25] & 28 & $\mathrm{OOK}$ & LO-based & $3.5-4.5$ & 27.24 & 175 & 14 & 0.095 & 3.8 \\
\hline JSSC '16 [26] & 65 & QPSK & LO-based & $6.25-8.5$ & 1000 & 175 & 221 & 0.7 & 442 \\
\hline TCASII '15 [27] & 65 & PPM+DB-BPSK & Edge combining & $3.1-4.8$ & 200 & 250 & 30 & 0.182 & 10.92 \\
\hline TBCAS '15 [28] & 90 & OOK & Edge combining & $3-5$ & 67 & 255 & 30 & 0.061 & 3.58 \\
\hline This work & 180 & OOK & LO-based & $3-6$ & 200 & 130 & 20 & 0.012 & 0.92 \\
\hline
\end{tabular}

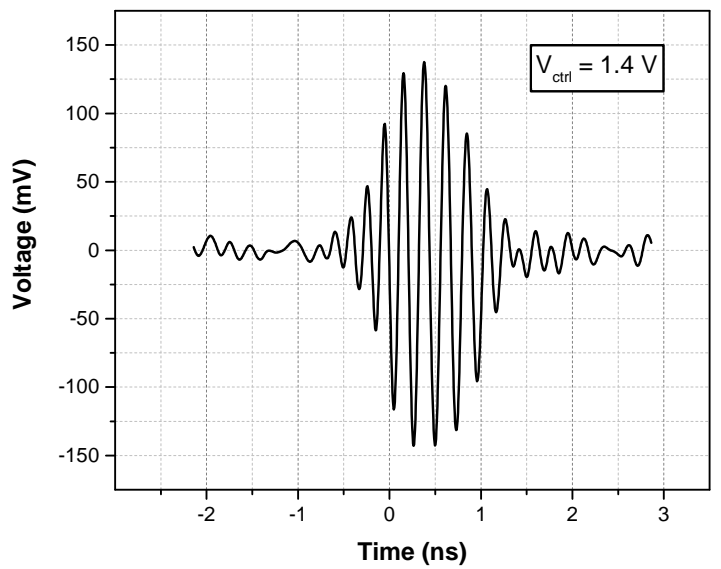

(a)

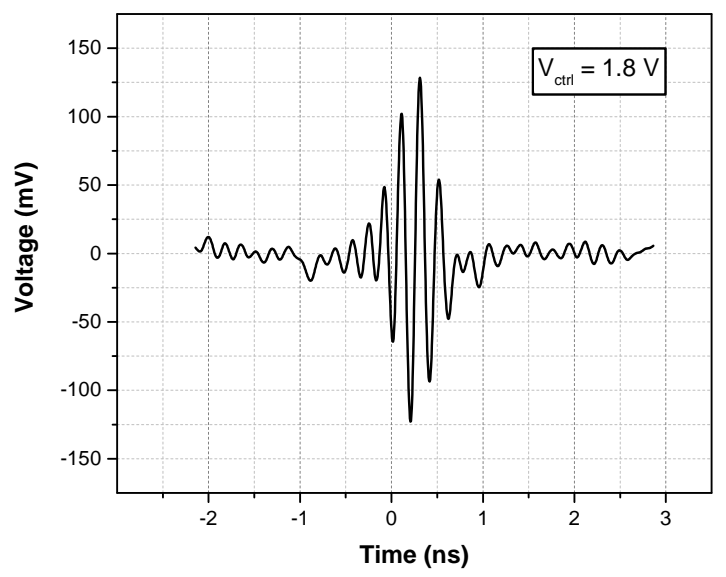

(b)

Fig. 6. IR-UWB pulse waveforms measured at the transmitter output while (a) $V_{\text {ctrl }}=1.4 \mathrm{~V}$ and (b) $V_{\text {ctrl }}=1.8 \mathrm{~V}$.

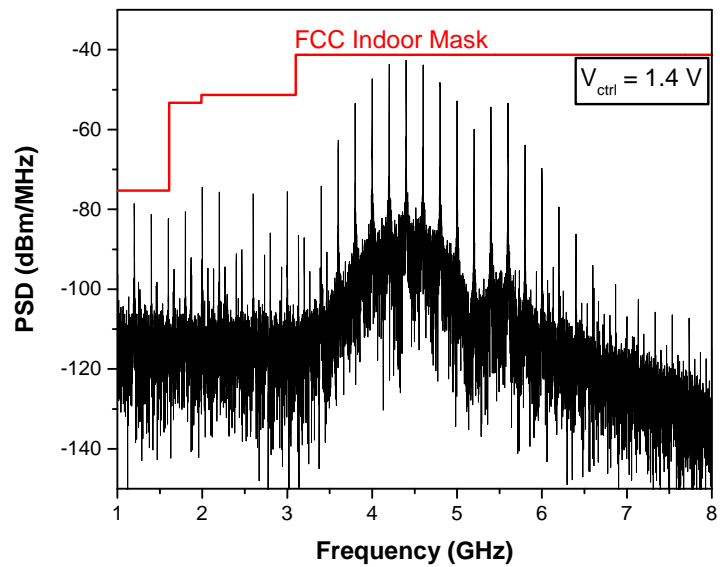

(a)

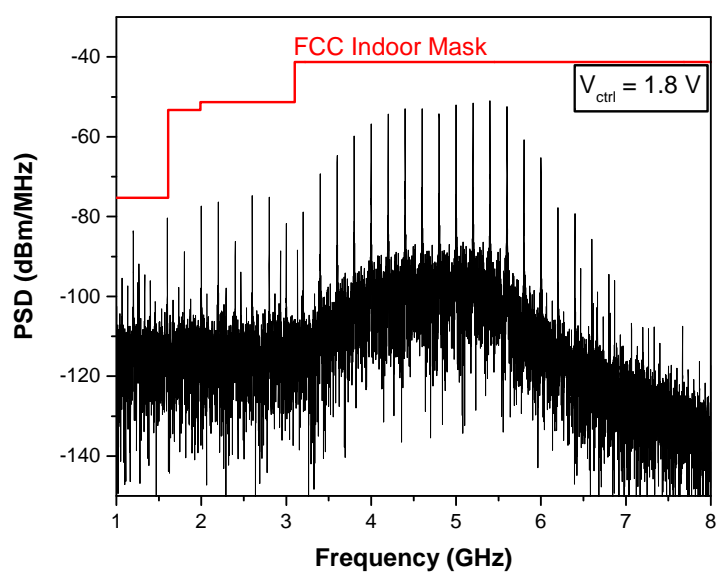

(b)

Fig. 7. PSD of the proposed transmitter at a data rate of $200 \mathrm{Mbps}$ while (a) $V_{c t r l}=1.4 \mathrm{~V}$ and (b) $V_{c t r l}=1.8 \mathrm{~V}$. 
marized and compared with recently published works in Table II. In addition to the energy consumed per bit, a normalized energy metric, which also covers the area occupation and peakto-peak output voltage, is introduced. The main contribution of this work is the minimization of the occupied area by using an active inductor-based LO while maintaining a low power consumption and high data rate communication.

\section{CONCLUSION}

An area and power efficient IR-UWB transmitter has been presented for high data rate short-range communication applications. The short pulses are generated by turning ON and OFF an oscillator. The oscillator is realized by an active inductor and a cross-coupled pair of transistors. The pulse generator is based on logic gates and determines the duration of the oscillation by modulating the bias current. Two single stage amplifiers are utilized for driving a differential $100 \Omega$ antenna load. The proposed integrated IR-UWB transmitter is fabricated in a UMC $180 \mathrm{~nm}$ CMOS process and occupies a die area of $0.012 \mathrm{~mm}^{2}$. Measurements show that the design dissipates a minimum of $20 \mathrm{pJ} / \mathrm{b}$ while transmitting at a $200 \mathrm{Mbps}$ data rate. It is demonstrated that high area consumption of spiral inductors in oscillators may be eliminated while maintaining FCC-compliant UWB operation.

\section{REFERENCES}

[1] M. Rezaei, E. Maghsoudloo, C. Bories, Y. De Koninck, and B. Gosselin, "A fully implantable multichip neural interface with a new scalable current-reuse front-end," in New Circuits and Systems Conference (NEWCAS), 2017 15th IEEE International. IEEE, 2017, pp. 365-368.

[2] H. Kassiri, S. Tonekaboni, M. T. Salam, N. Soltani, K. Abdelhalim, J. L. P. Velazquez, and R. Genov, "Closed-loop neurostimulators: A survey and a seizure-predicting design example for intractable epilepsy treatment," IEEE transactions on biomedical circuits and systems, vol. 11, no. 5, pp. 1026-1040, 2017.

[3] E. B. Assi, D. K. Nguyen, S. Rihana, and M. Sawan, "Towards accurate prediction of epileptic seizures: A review," Biomedical Signal Processing and Control, vol. 34, pp. 144-157, 2017.

[4] S. B. Lee, B. Lee, M. Kiani, B. Mahmoudi, R. Gross, and M. Ghovanloo, "An inductively-powered wireless neural recording system with a charge sampling analog front-end," IEEE sensors journal, vol. 16, no. 2, pp. 475-484, 2016.

[5] C. Carboni, L. Bisoni, N. Carta, R. Puddu, S. Raspopovic, X. Navarro, L. Raffo, and M. Barbaro, "An integrated interface for peripheral neural system recording and stimulation: system design, electrical tests and in-vivo results," Biomedical microdevices, vol. 18, no. 2, p. 35, 2016.

[6] X. Zou, L. Liu, J. H. Cheong, L. Yao, P. Li, M.-Y. Cheng, W. L. Goh, R. Rajkumar, G. S. Dawe, K.-W. Cheng et al., "A 100-channel 1-mw implantable neural recording ic," IEEE Transactions on Circuits and Systems I: Regular Papers, vol. 60, no. 10, pp. 2584-2596, 2013.

[7] S. Kim, P. Tathireddy, R. A. Normann, and F. Solzbacher, "Thermal impact of an active 3-d microelectrode array implanted in the brain," IEEE Transactions on Neural Systems and Rehabilitation Engineering, vol. 15, no. 4, pp. 493-501, 2007.

[8] M. Ballini, J. Müller, P. Livi, Y. Chen, U. Frey, A. Stettler, A. Shadmani, V. Viswam, I. L. Jones, D. Jäckel et al., "A 1024-channel cmos microelectrode array with 26,400 electrodes for recording and stimulation of electrogenic cells in vitro," IEEE journal of solid-state circuits, vol. 49, no. 11, pp. 2705-2719, 2014

[9] R. Muller, H.-P. Le, W. Li, P. Ledochowitsch, S. Gambini, T. Bjorninen, A. Koralek, J. M. Carmena, M. M. Maharbiz, E. Alon et al., "A minimally invasive 64-channel wireless $\mu$ ecog implant," IEEE Journal of Solid-State Circuits, vol. 50, no. 1, pp. 344-359, 2015.

[10] J. Tan, W.-S. Liew, C.-H. Heng, and Y. Lian, "A 2.4 ghz ulp reconfigurable asymmetric transceiver for single-chip wireless neural recording ic," IEEE transactions on biomedical circuits and systems, vol. 8, no. 4, pp. 497-509, 2014.
[11] H. Kassiri, A. Bagheri, N. Soltani, K. Abdelhalim, H. M. Jafari, M. T. Salam, J. L. P. Velazquez, and R. Genov, "Battery-less triband-radio neuro-monitor and responsive neurostimulator for diagnostics and treatment of neurological disorders," IEEE Journal of Solid-State Circuits, vol. 51, no. 5, pp. 1274-1289, 2016.

[12] K. Ture, R. Ranjandish, G. Yilmaz, S. Seiler, H. R. Widmer, A. Schmid, F. Maloberti, and C. Dehollain, "Power/data platform for high data rate in implanted neural monitoring system," in Biomedical Circuits and Systems Conference (BioCAS), 2017 IEEE. IEEE, 2017.

[13] M. Kiani and M. Ghovanloo, "A 20-mb/s pulse harmonic modulation transceiver for wideband near-field data transmission," IEEE Transactions on Circuits and Systems II: Express Briefs, vol. 60, no. 7, pp. 382-386, 2013.

[14] S. Bourdel, Y. Bachelet, J. Gaubert, R. Vauche, O. Fourquin, N. Dehaese, and $\mathrm{H}$. Barthelemy, "A 9-pj/pulse 1.42-vpp ook cmos uwb pulse generator for the 3.1-10.6-ghz fcc band," IEEE Transactions on Microwave Theory and Techniques, vol. 58, no. 1, pp. 65-73, 2010.

[15] S. A. Mirbozorgi, H. Bahrami, M. Sawan, L. A. Rusch, and B. Gosselin, "A single-chip full-duplex high speed transceiver for multi-site stimulating and recording neural implants," IEEE transactions on biomedical circuits and systems, vol. 10, no. 3, pp. 643-653, 2016.

[16] P. P. Mercier, D. C. Daly, and A. P. Chandrakasan, "An energy-efficient all-digital uwb transmitter employing dual capacitively-coupled pulseshaping drivers," IEEE Journal of Solid-State Circuits, vol. 44, no. 6, pp. 1679-1688, 2009.

[17] Y.-J. Lin, S.-Y. Park, X. Chen, D. Wentzloff, and E. Yoon, "4.32-pj/b, overlap-free, feedforward edge-combiner-based ultra-wideband transmitter for high-channel-count neural recording," IEEE Microwave and Wireless Components Letters, vol. 28, no. 1, pp. 52-54, 2018.

[18] S. V. Mir-Moghtadaei, A. Fotowat-Ahmady, A. Z. Nezhad, and W. A. Serdijn, "A 90 nm-cmos ir-uwb bpsk transmitter with spectrum tunability to improve peaceful uwb-narrowband coexistence," IEEE Transactions on Circuits and Systems I: Regular Papers, vol. 61, no. 6, pp. 18361848, 2014.

[19] N.-S. Kim and J. M. Rabaey, "A high data-rate energy-efficient triplechannel uwb-based cognitive radio," IEEE Journal of Solid-State Circuits, vol. 51, no. 4, pp. 809-820, 2016.

[20] J. Craninckx and M. Steyaert, "Low-noise voltage-controlled oscillators using enhanced lc-tanks," IEEE Transactions on Circuits and Systems II: Analog and Digital Signal Processing, vol. 42, no. 12, pp. 794-804, 1995.

[21] L.-H. Lu, H.-H. Hsieh, and Y.-T. Liao, "A wide tuning-range cmos vco with a differential tunable active inductor," IEEE Transactions on Microwave Theory and Techniques, vol. 54, no. 9, pp. 3462-3468, 2006.

[22] N. M. Nguyen and R. G. Meyer, "Start-up and frequency stability in high-frequency oscillators," IEEE Journal of Solid-State Circuits, vol. 27, no. 5, pp. 810-820, 1992.

[23] S. Diao, Y. Zheng, and C.-H. Heng, "A cmos ultra low-power and highly efficient uwb-ir transmitter for wpan applications," IEEE Transactions on Circuits and Systems II: Express Briefs, vol. 56, no. 3, pp. 200-204, 2009.

[24] P. Gunturi, N. W. Emanetoglu, and D. E. Kotecki, "A 250-mb/s data rate ir-uwb transmitter using current-reused technique," IEEE Transactions on Microwave Theory and Techniques, vol. 65, no. 11, pp. 4255-4265, 2017.

[25] G. de Streel, F. Stas, T. Gurné, F. Durant, C. Frenkel, A. Cathelin, and D. Bol, "Sleeptalker: A ulv 802.15. 4a ir-uwb transmitter soc in 28$\mathrm{nm}$ fdsoi achieving $14 \mathrm{pj} / \mathrm{b}$ at $27 \mathrm{mb} / \mathrm{s}$ with channel selection based on adaptive fbb and digitally programmable pulse shaping," IEEE Journal of Solid-State Circuits, vol. 52, no. 4, pp. 1163-1177, 2017.

[26] J. Ko and R. Gharpurey, "A pulsed uwb transceiver in $65 \mathrm{~nm}$ cmos with four-element beamforming for 1 gbps meter-range wpan applications," IEEE Journal of Solid-State Circuits, vol. 51, no. 5, pp. 1177-1187, 2016.

[27] K. Na, H. Jang, H. Ma, Y. Choi, and F. Bien, "A 200-mb/s data rate 3.1-4.8-ghz ir-uwb all-digital pulse generator with db-bpsk modulation," IEEE Transactions on Circuits and Systems II: Express Briefs, vol. 62, no. 12 , pp. $1184-1188,2015$.

[28] A. Ebrazeh and P. Mohseni, " 30 pj/b, $67 \mathrm{mbps}$, centimeter-to-meter range data telemetry with an ir-uwb wireless link," IEEE transactions on biomedical circuits and systems, vol. 9, no. 3, pp. 362-369, 2015. 